\title{
Acceleration Sensitivity of Small-Gap Capacitive Micromechanical Resonator Oscillators
}

\author{
Bongsang Kim ${ }^{1,2}$, Mehmet Akgul ${ }^{1}$, Yang Lin ${ }^{1}$, Wei-Chang Li ${ }^{1}$, Zeying Ren ${ }^{1}$, and Clark T.-C. Nguyen ${ }^{1}$ \\ ${ }^{1}$ Department of Electrical Engineering and Computer Sciences \\ University of California, Berkeley \\ Berkeley, California, 94720, United States of America \\ ${ }^{2}$ Sandia National Laboratories \\ Albuquerque, New Mexico, 87123, United States of America
}

bongsang@gmail.com

\begin{abstract}
The vector components of acceleration sensitivity $\Gamma$ for a closed-loop oscillator referenced to a wine-glass disk arraycomposite resonator employing tiny $(\sim 92 \mathrm{~nm})$ electrode-toresonator capacitive transducer gaps were measured along axes perpendicular and parallel to the substrate to be $\Gamma_{\text {verticar }} \sim 13.6 \mathrm{ppb} / \mathrm{g}$ and $\Gamma_{\text {laterar }}-4.92 \mathrm{ppb} / \mathrm{g}$, respectively, which are on par with commercial quartz-based oscillator products. Interestingly, the measured acceleration sensitivity greatly exceeds the prediction of theory. In particular, models for frequency shifts due to variations in electrical stiffness and mechanical stress predict acceleration sensitivities orders of magnitude lower than measured here. Consideration of other microphonic contributors reveals that the measurements of this work were probably limited by the bond wires and package stresses of the board-level realization of the oscillator, so are very likely not representative of the performance actually achievable by a fullyintegrated micromechanical resonator oscillator, where MEMS and transistors share a single chip. Still, the measured microphonic performance on par with mid-grade quartz oscillators at least provides some reassurance that the tiny electrode-toresonator gaps used in high frequency capacitively transduced micromechanical resonators will not compromise the stabilities of oscillators referenced to them in conventional applications that currently accept mid-grade quartz resonators.
\end{abstract}

Keywords-MEMS, microphonics, acceleration sensitivity, vibration, capacitive transducer, small gap, electrical stiffness.

\section{INTRODUCTION}

Micromechanical resonators constructed via MEMS technology have recently been spotlighted as potential next generation mechanical signal processors for use in oscillators, filters, mixers, and even amplifiers. Beyond offering substantial reductions in size, power consumption, and manufacturing cost, their potential for single-chip integration with CMOS integrated circuits is expected to yield a degree of system-level miniaturization and resultant parasitic loss reductions that encourage a paradigm shift in wireless communication system architecture [1]. Indeed, capacitively transduced micromechanical resonators, such as shown in Fig. 1, that use voltageinduced electric fields across electrode-to-resonator gaps to excite and sense resonance have now been demonstrated with long-term stability less than \pm 2 ppm over 10,000 hours of operation [2]; excellent temperature stability of better than \pm 0.05 ppm over $-20^{\circ} \mathrm{C} \sim 80^{\circ} \mathrm{C}$ using less than $18 \mathrm{~mW}$ of oven power [3]; quality factor $Q$ greater than 10,000 at $\mathrm{GHz}$ frequencies [4], and $f \cdot Q$ products exceeding $5.1 \times 10^{13}$ [5]. Moreover, the recent demonstration of phase-noise performance that meets commercial global systems for mobile communication (GSM) specifications suggests that micromechanical resonators should soon be usable in higher-end applications beyond consumer electronics, where micromechanical resonator-based oscillators are already commercialized and now encroaching on markets traditionally dominated by quartz [6].

While the use of capacitive transduction provides many benefits, including higher $Q$ and a larger available set of materials, early capacitive transducer realizations suffered from a relatively weaker electromechanical coupling factor compared to other transduction methods. The resulting high motional
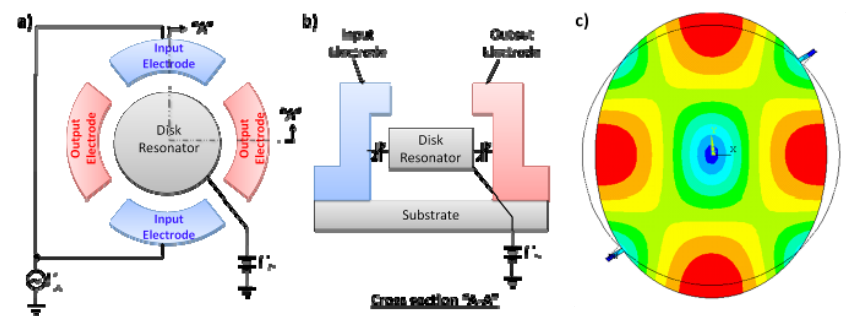

Fig. 1 (a) Top view schematic and (b) cross-section of a typical disk-type capacitively transduced micromechanical resonator. Voltage-induced electric fields across electrode-to-resonator gaps are used to excite and sense the resonator. c) ANSYS finite element simulated mode-shape.

This work was supported by the DARPA N/MEMS S\&T program. 


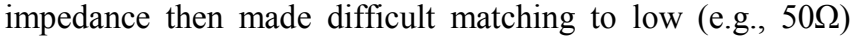
impedance systems. Among methods for improving electromechanical coupling in capacitive transducers, reducing the electrode-to-resonator gap spacing is mathematically the most effective [7]. Doing so, however, is not a simple prospect when the gaps are lateral ones, such as in Fig. 1. For example, although e-beam lithography and subsequent etching to form lateral gaps has been suggested as one possible way to reduce gaps over those obtainable via conventional lithography [8], its low throughput and high cost pose significant barriers to volume production. As a result, sidewall sacrificial spacer methods, such as first used in [9], have dominated among methods for achieving lateral gaps down to $50 \mathrm{~nm}$, but below this suffer from etchant and etch by-product diffusion limitations that compromise yield. The latest remedy to this uses atomic layer deposition (ALD) to deposit high-k dielectric material into a sacrificial spacer-defined gap, partially (but not fully) filling it to achieve a much smaller effective gap spacing [7]. This approach recently achieved repeatable gap spacings as small as $37 \mathrm{~nm}$ that enabled the first micromechanical resonators with simultaneous high $Q(>70,000)$ and low motional impedance $\left(R_{x}<130 \Omega\right)$ at $61 \mathrm{MHz}[10]$.

But gap reduction also raises concerns regarding susceptibility to acceleration, i.e., to microphonics. Indeed, many oscillators find use in dynamic platforms, for which resilience against vibration is important. Acceleration from environmental vibration is well known to degrade the short-term stability of oscillators, especially ones referenced to mechanical resonators that respond to acceleration by shifts in resonance frequency. Needless to say, acceleration sensitivity has been studied extensively in quartz-crystal oscillators [11].

The acceleration-induced resonance frequency shift $\Delta f$ for a mechanical resonator is often expressed as,

$$
\frac{\Delta f}{f}=\bar{\Gamma} \bullet \bar{a} \quad \text { with } \quad \bar{\Gamma}=\Gamma_{x} \hat{x}+\Gamma_{y} \hat{y}+\Gamma_{z} \hat{z}
$$

where $f$ is the resonance frequency, $\bar{a}$ is the acceleration in vector form, and $\bar{\Gamma}$ is the acceleration sensitivity of the resonator gauging the amount of the frequency shift for a given acceleration.

If an oscillator with a mechanical reference resonator experiences a vibration at frequency $f_{v}$, its output signal phase is modulated by the instantaneous frequency shift of the resonator governed by (1), creating sideband peaks in the output power spectrum as shown in the schematic in Fig. 2. Since only power at the carrier frequency constitutes the output of an oscillator, and power at all other frequencies is considered noise, these side peaks can be interpreted as noise at a frequency offset of $f_{v}$ from the carrier, and their relative height can be expressed as

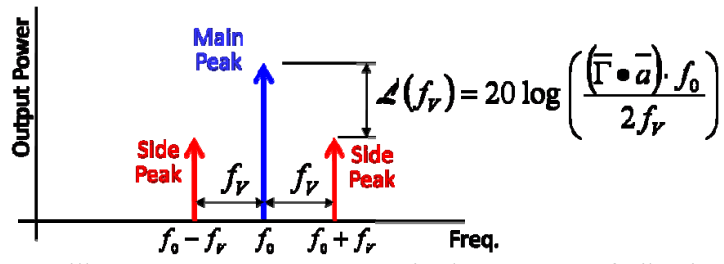

Fig. 2: Oscillator output power spectrum in the presence of vibration with frequency $f_{v}$. Sideband peaks appear at offsets $f_{v}$ from the carrier frequency.

$$
L\left(f_{V}\right)=20 \log \left(\frac{(\bar{\Gamma} \bullet \bar{a}) \cdot f}{2 f_{v}}\right)
$$

It should be noted that, in the presence of random vibration, this noise would appear as $1 / f^{2}$ noise, and would therefore dominate the oscillator phase noise performance. Indeed, resonator acceleration sensitivity often governs oscillator performance in dynamic platforms.

Recently, the acceleration sensitivities of oscillators referenced to capacitively transduced micromechanical resonators with gaps of $1 \mu \mathrm{m}$ and $150 \mathrm{~nm}$ were measured and reported [12, 13]. This work focuses on smaller-gapped capacitive micromechanical resonator oscillators, with an aim to elucidate the fundamental mechanisms governing the acceleration sensitivity of micromechanical resonators that employ electrode-toresonator gaps with spacings less than $100 \mathrm{~nm}$. Two main mechanisms are considered: 1) changes in electrical stiffness caused by acceleration-induced changes in electrode-toresonator gap spacing; and 2) the effect of accelerationinduced stress on the resonant structure. Using ANSYS finite element simulation, silicon surface micromachined resonators are modeled and analyzed, and these models are compared with measurement data. Other potential mechanisms associated with the testing apparatus that can dominate the acceleration sensitivity of tested resonator oscillators are discussed, as well.

\section{Potential CONTRIBUtORS TO ACCELERATION SENSITIVITY}

\section{A. Acceleration-Induced Electrical Stiffness Changes}

Spring softening due to electrical stiffness is a well known non-linear dynamical phenomenon associated with capacitive transduction. In capacitive transduction, as described in Fig. 3, the electrical drive force generated by a constant bias voltage $V_{P}$ applied across the electrode-to-resonator gap increases as the resonator approaches the electrode, i.e., as the capacitive gap decreases, and decreases as the resonator moves farther from the electrode. Since the change in force is proportional to displacement, it can be modeled as an effective electrical stiffness that can be expressed as

$$
k_{e}=\frac{\partial F_{e}}{\partial x}=\frac{\varepsilon A V_{P}^{2}}{d^{3}}
$$

where $\varepsilon$ and $A$ are the permittivity and overlap area, respec-

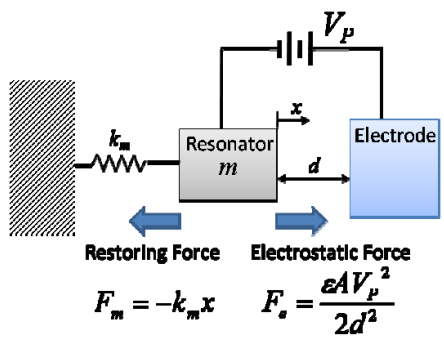

Fig. 3: Lumped model of a capacitively transduced micromechanical resonator. The electrostatic force depends on the gap size between the resonator and the electrode; as the resonator displacement $x$ increases, the gap size decreases resulting in an increase in the electrostatic force. This displacement dependency of the electrostatic force behaves similarly to a mechanical restoring force, but in the opposite direction. 
tively, of the electrode-to-resonator gap. Since it acts against the restoring force of the resonator's mechanical stiffness, $k_{e}$ subtracts from the overall stiffness of the resonator structure, yielding a resonance frequency given by

$$
f_{r}=\frac{1}{2 \pi} \sqrt{\frac{k}{m}}=\frac{1}{2 \pi} \sqrt{\frac{k_{m}-k_{e}}{m}}=f_{o} \sqrt{1-\frac{k_{e}}{k_{m}}}
$$

where $k$ is the overall stiffness, $m$ is the resonator mass, $k_{m}$ is the mechanical stiffness, and $f_{o}$ is the original natural frequency of the resonator (with $V_{P}=0 \mathrm{~V}$ ).

Using (3) and (4), the fractional frequency change of the resonance frequency brought about by acceleration-induced changes in gap spacing and overlap area can be expressed as

$$
\begin{array}{ll}
\frac{\Delta f}{f}=\frac{1}{2} \frac{\varepsilon V_{P}^{2}}{d^{4} k_{m}} \Delta d & \text { for gap spacing change } \\
\frac{\Delta f}{f}=-\frac{1}{2} \frac{\varepsilon V_{P}^{2}}{d^{3} k_{m}} \Delta A & \text { for overlap area change }
\end{array}
$$

where $\Delta d$ is change in gap spacing and $\Delta A$ is change in overlap area. Note that as the electrode-to-resonator gap $d$ shrinks, (5) predicts much larger shifts in frequency shifts due to acceleration-induced shifts in both cases.

\section{B. Acceleration-Induced Stress Changes}

As a mechanical resonator experiences acceleration, a stress gradient is induced in the resonant structure, which results in a shift in resonance frequency. Reference [12] investigated this stress effect for the case of beam-type MEMS, for which the principal mechanism for acceleration sensitivity is axial stress due to the D'Alembert force from a floating coupled mass. This magnitude of this effect depends on the magnitude of the induced force, making the resonator mass an important factor. In other words, as mass increases, acceleration induces more force, which then generates a larger shift in the resonance frequency. In addition, the stress distribution, i.e., locations of highest stress, also influence the amount of frequency shift. Finite element simulation is an effective means for modeling such acceleration induced-stress distributions on a resonant structure and for linking them to the resulting resonance frequency change.

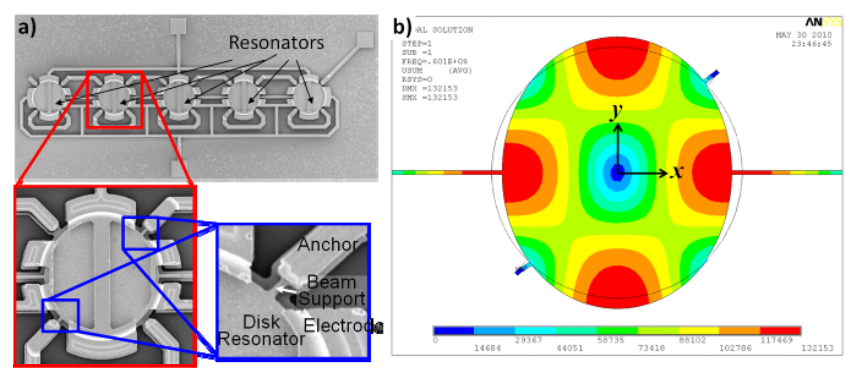

Fig. 4: a) SEM images of the capacitively transduced micromechanical resonators used in this study. Five resonators are coupled in parallel via halfwave beam, and each disk is suspended from the substrate by two diagonally anchored support beams $(4.5 \mu \mathrm{mL} \times 1 \mu \mathrm{mW} \times 3 \mu \mathrm{mH})$ at the vibration nodes. The $92 \mathrm{~nm}$ electrode-to-resonator gap is defined by an $\mathrm{SiO}_{2}$ sidewall sacrificial layer. b) Mode shape (wine-glass mode) of an individual disk from ANSYS FE simulation. At resonance, all disk resonators vibrate identically.

\section{Vibration Susceptibility of Non-Resonant Components}

Many microwave electronic components are known to be sensitive to vibration, from coaxial cables that typically exhibit acceleration sensitivities from $10^{-4} \sim 10^{-6} \mathrm{rad} / \mathrm{g}$ [14]; to bandpass filters; mechanical phase shifters; and printed circuit boards [15]. Indeed, investigations on the role of such components on oscillator acceleration sensitivity $[14,16]$ reveal that they can often dominate. Thus, the resolution of any boardlevel test set-up gauging the acceleration sensitivity of the micromechanical resonator oscillators of interest, here, is ultimately limited by contributions from non-resonant boardlevel components.

\section{MODELING}

To investigate the acceleration sensitivity of small-gap capacitively transduced micromechanical resonators, this work employs the polysilicon wine-glass mode disk arraycomposite resonator depicted in Fig. 4. Here, five disk resonators are coupled by half-wavelength beams to form a composite resonator structure that vibrates at a designed mode frequency. Because it uses several resonators, this composite resonator handles more power and exhibits lower impedance than a single stand-alone resonator, both of which are beneficial to oscillator design and performance. Each disk has a radius of $R=32 \mathrm{um}$ and thickness of $h=3 \mathrm{um}$, and is suspended by two support beams attached opposite one another at wineglass mode nodal points, as indicated in Fig. 4.

When $n$ resonators are assembled into an array-composite, the composite structure takes on values of stiffness and mass that are $n$ times the value at the same location on a single stand-alone resonator. In other words, the composite structure is $n$ times stiffer than any one of its constituent resonators. However, the mass of the composite is also larger, so forces generated by accelerations will likely also be approximately $n$ times larger, suggesting that the acceleration response of the composite should be similar to that of a single resonator, although not exactly the same. In order to simplify the analysis so as to more easily delineate important dependencies, the analyses to follow are done on a single resonator, with the understanding that they will only be approximate for the array-composite actually tested.

Using models from [17], the mechanical stiffness at the anti-nodes of a stand-alone constituent disk resonator in Fig. 4 is calculated to be $k_{m}=6.61 \times 10^{5} \mathrm{~N} / \mathrm{m}$. The capacitive transducer area for the disk is $A=5.04 \times 10^{10} \mathrm{~m}^{2}$. The resonance frequency is $f=61 \mathrm{MHz}$, and the sacrificial sidewall spacer-defined [18] electrode-to-resonator gap is $d=92 \mathrm{~nm}$. At $V_{P}=12 \mathrm{~V}$, using (3), the electrical stiffness is calculated to be $k_{e}=885 \mathrm{~N} / \mathrm{m}$. These values are used to obtain some of the numbers in the analyses to follow.

\section{A. Acceleration-Induced Electrical Stiffness Changes}

\section{1) Vertical Acceleration}

When a disk resonator experiences a vertical (or $z$ directed) acceleration, the resonator displaces in that direction, yielding a change in transducer area $A$, as shown in Fig. 5. The resulting change in electrical stiffness, $k_{e}$, can be expressed as 
a)
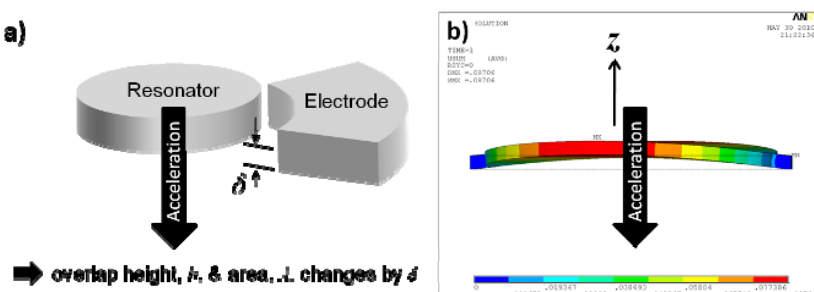

Fig. 5: a) Conceptual schematic of a capacitively transduced micromechanical resonator experiencing vertical acceleration. The capacitive transducer area changes due to vertical displacement of the resonator. b) Side view of ANSYS finite element simulation showing resonator displacement due to vertical acceleration.

$$
\Delta k_{e}=\frac{\varepsilon A V_{P}^{2}}{d^{3}} \frac{\Delta A}{A}=k_{e} \frac{\Delta A}{A}
$$

The fractional transducer area change, $\Delta A / A$, can be approximated as the resonator displacement, $\delta$, with respect to the resonator thickness, $h$, after which equation (6-1) can be expressed as,

$$
\Delta k_{e}=k_{e} \frac{\delta}{h}
$$

Using ANSYS finite element modeling, the resonator displacement per unit acceleration in the $z$-direction is simulated to be $\delta=8.71 \times 10^{5} \mathrm{~nm} / \mathrm{g}$. Using this in (1), (5), and (6-2), the $z$ direction acceleration sensitivity for a capacitively transduced micromechanical disk resonator becomes

$$
\Gamma_{Z, \text { elec.stiff }}=1.81 \times 10^{-11} / \mathrm{g}
$$

\section{2) Lateral Acceleration}

When a disk experiences a lateral (i.e., in plane) acceleration, its transducer gap, $d$, changes as shown in Fig. 6a. Unlike the case of vertical acceleration, since the resonator is anchored diagonally, its displacement varies depending on the orientation of the acceleration. As shown in the ANSYS finite element simulation of Fig. $6 \mathrm{~b}$, the resonator displacement is maximized when the acceleration is perpendicular to the anchors and bends the supporting beams flexurally. In contrast, when the acceleration is parallel to the anchors, it must act against substantially stiffer restoring forces that minimize displacements along this direction, thus, minimizing the acceleration sensitivity. Fig. 7 presents plots of $x$ and $y$ direction resonator displacements $\left(\delta_{X}\right.$ and $\left.\delta_{Y}\right)$ with respect to the orientation of the acceleration. The directional dependence of resonator displacements in units of nano-meter per $g$ can be expressed as a)

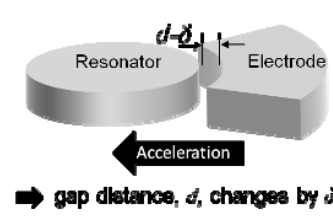

b)

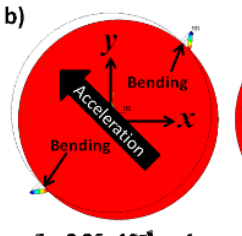

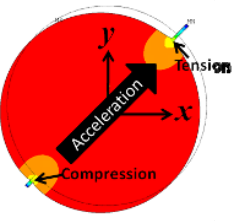

$-1.71 \times 10^{\mathrm{H}} \mathrm{mas} / \mathrm{g}$
Fig. 6: a) Conceptual schematic of a capacitively transduced micromechanical resonator experiencing lateral acceleration. Here, the capacitive transducer gap changes due to the lateral displacement of the resonator. b) Finite element simulation of resonator displacement due to the lateral acceleration. When the acceleration orientation is perpendicular to the anchors (left side), the support beams bend, generating more displacement than when the acceleration is parallel to the anchors (right side).

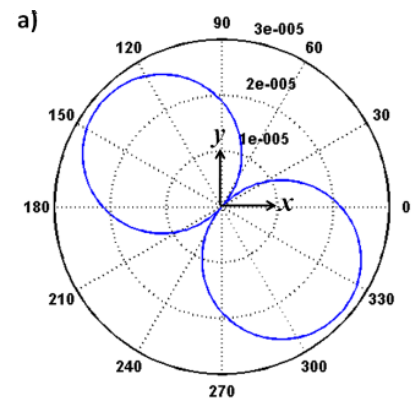

X-Direction Displacement, $\delta,(\mathrm{nm} / \mathrm{g})$ vs. Acceleration Orientation $\left({ }^{\circ}\right)$

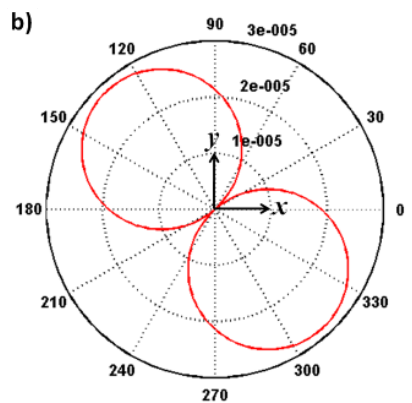

Y-Direction Displacement, $\delta$. $(\mathrm{nm} / \mathrm{g})$ vs. Acceleration Orientation ( $\left(^{\circ}\right)$
Fig. 7: Plots of resonant structure displacement with respect to acceleration orientation. a) $x$-direction displacement per $1 \mathrm{~g}$ acceleration and b) $y$-direction displacement per $1 \mathrm{~g}$ acceleration in nano-meters. a)

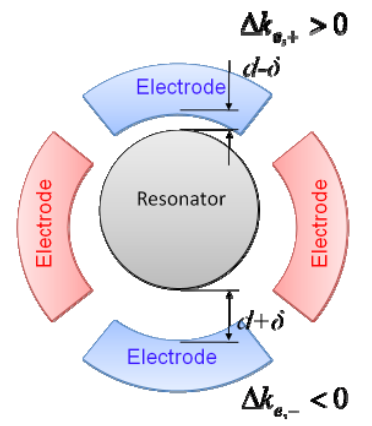

b)

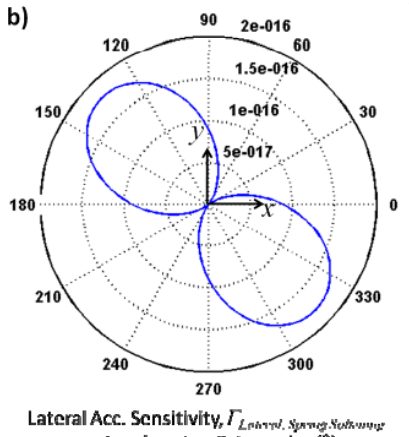

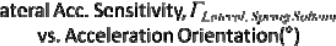

Fig. 8: a) When the resonator displaces, the transducer gap decreases by $\delta$ on one side (thus $k_{e}$ increases) at the same time the gap increases by $\delta$ on the other (thus $k_{e}$ decreases). The combined electrical stiffness change thus cancel to first order, resulting in a much smaller change in total resonator stiffness. This, of course, enhances the resonator's resilience to against lateral direction acceleration. b) Plot of lateral acceleration sensitivity due to electrical stiffness changes with respect to the orientation of the acceleration. When the resonator sees accelerations perpendicular to its support beams, its acceleration sensitivity maximizes at $\Gamma_{\text {Lateral, elec.stiff }}=1.80^{-16} / \mathrm{g}$.

$$
\begin{aligned}
& \delta_{X}=1.37 \times 10^{-5} a_{X}-1.51 \times 10^{-5} a_{Y} \\
& \delta_{Y}=-1.51 \times 10^{-5} a_{X}+1.37 \times 10^{-5} a_{Y}
\end{aligned}
$$

For lateral accelerations, the overall change in electrical stiffness becomes more complicated when electrodes are placed symmetrically around the resonator, as is the case for the wine-glass disk under consideration. Fig. 8a shows an example case when the disk displaces in the $y$-direction. Here, the electrode-to-resonator gap decreases by $\delta$ on the upper side, but increases by $\delta$ on the lower side. As a result, $k_{e}$ increases at the upper side, but decreases at the lower side. The overall resonator stiffness contribution from electrical stiffness can thus be expressed as the difference between these two:

$$
\begin{aligned}
\Delta k_{e} & =\Delta k_{e,+}-\Delta k_{e--} \\
& =6 \frac{\varepsilon(A / 4) V_{P}^{2}}{d^{3}}\left(\frac{\delta}{d}\right)^{2}=\frac{3}{2} k_{e}\left(\frac{\delta}{d}\right)^{2}
\end{aligned}
$$

Combining (8) and (9), expressions for lateral acceleration sensitivity can be expressed as

$$
\begin{aligned}
& \Gamma_{X, \text { elec.stiff }}=-1.27 \times 10^{-16} / \mathrm{g} \\
& \Gamma_{Y, \text { elec.stiff }}=1.27 \times 10^{-16} / \mathrm{g}
\end{aligned}
$$




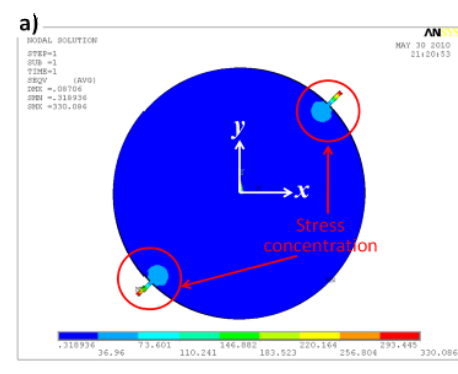

$\Delta / f--2.021 \times 10^{15} a$

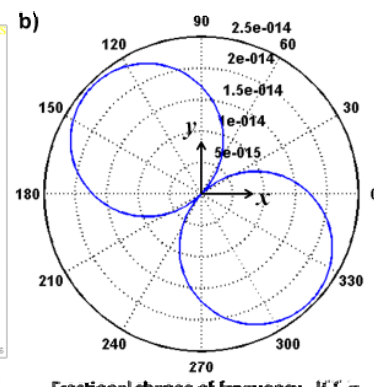

Fractional change of frequency, $y f g$ พ. Acceleration Crientationt

Fig. 9: a) ANSYS FE simulation to model resonance frequency changes caused by acceleration induced stress. Most of the induced stress is concentrated locally around the vibration nodes, and thus, have very little influence on resonance contortions. b) Plot of lateral acceleration sensitivity due to acceleration-induced stress with respect to the acceleration orientation. When the resonator experiences acceleration perpendicular to the support beams, its acceleration sensitivity maximizes at $\Gamma_{\text {Lateral, stress }}=2.43^{-14} / \mathrm{g}$.

or

$$
\begin{aligned}
& \Gamma_{\text {perpendiculartoanchors, elec.stiff }}=-1.80 \times 10^{-16} / \mathrm{g} \\
& \Gamma_{\text {parelleltoanchors, elec.stiff }}=-6.48 \times 10^{-19} / \mathrm{g}
\end{aligned}
$$

and plotted as shown in Fig. 8b. Note that the predicted lateral direction acceleration sensitivities of (9) are substantially smaller than the vertical direction sensitivities modeled in (7) by several orders of magnitude. Clearly, cancellation of electrical stiffness contributions by symmetrical electrode placement is an effective means for nulling electrical-stiffnessbased acceleration sensitivity in the lateral direction.

\section{B. Acceleration-Induced Stress Changes}

The change in resonance frequency due to accelerationinduced stress changes was explored by means of ANSYS finite element simulation, as shown in Fig. 9. Again, lateral acceleration sensitivity exhibits directionality with respect to the acceleration orientation, i.e. when the acceleration is perpendicular to the anchors maximum stress/strain is induced yielding the largest frequency shift, and when the acceleration is parallel to the anchors minimum stress/strain is induced, thus producing the least frequency shift. From the ANSYSsimulated plot in Fig. 9b, expressions for stress-based acceleration sensitivity for the resonance frequency of a wine-glass disk resonator can be summarized as

$$
\begin{aligned}
& \Gamma_{X, \text { stress }}=1.73 \times 10^{-14} / \mathrm{g} \\
& \Gamma_{Y, \text { stress }}=-1.71 \times 10^{-14} / \mathrm{g} \\
& \Gamma_{Z, \text { stress }}=-2.02 \times 10^{-15} / \mathrm{g}
\end{aligned}
$$

or

$$
\begin{aligned}
& \Gamma_{\text {perpendiculartoanchors, stress }}=-4.94 \times 10^{-14} / \mathrm{g} \\
& \Gamma_{\text {parelleltoanchors, stress }}=-2.64 \times 10^{-16} / \mathrm{g}
\end{aligned}
$$

It should be noted that the acceleration sensitivity values in (11) are several orders of magnitude smaller than those for tuning fork beam-type resonators reported in [12], which were $\Gamma_{\text {tuning fork }}=10^{-8} \sim 10^{-9} / \mathrm{g}$. This is because 1) the anchors for the wine-glass mode resonators in this study are strategically placed at vibration nodes, so that induced stresses are mostly local with very little impact on the rest of the resonant struc-
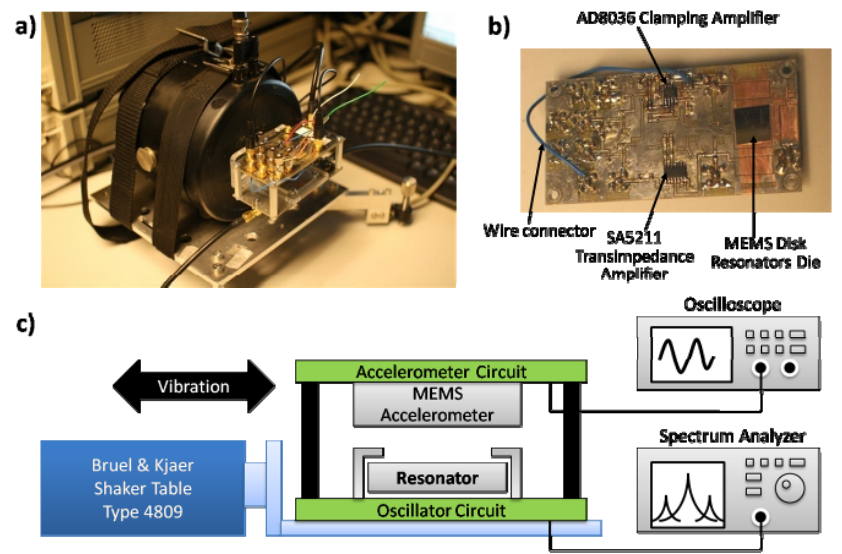

Fig. 10: a) Photo of the experimental setup. b) Oscillator PCB used in this study. Here, wires were used to reconfigure the PCB and resonators were connected to the circuit board via wire-bonds. c) Schematic of the experimental setup. When the circuit boards are excited, the acceleration amplitude and oscillator output power are measured simultaneously.

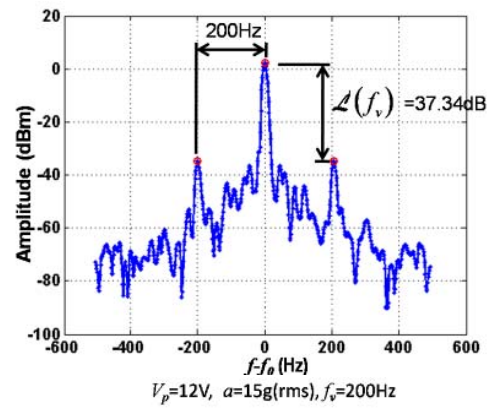

Fig. 11: Measured output power spectrum while the oscillator PCB experiences lateral direction acceleration at $f_{v}=200 \mathrm{~Hz}$.

ture, as can be seen in Fig. 9a; and 2) high-frequency disk resonators have smaller masses, so induce much less force than low frequency tuning forks under identical accelerations.

\section{MEASUREMENT}

Fig. 10 illustrates the experimental setup used to measure acceleration sensitivity of oscillators referenced to wine-glass disk array-composite resonators, such as shown in Fig. 4. The rudimentary sustaining oscillator used in these measurements consisted of an SA5211 transimpedance amplifier and an AD8322 clamping amplifier, and connected with the resonator die on the printed circuit board (PCB) shown in Fig. 10 b. Freescale accelerometers (MMA1213 and MMA3201) housed on another PCB were used to accurately measure acceleration. A Bruel \& Kjaer Type 4809 shaker, to which both PCB's were attached, provided accelerations while an Agilent 8711A spectrum analyzer measured the output power of the oscillator. Fig. 11 presents a measured plot of the oscillator output power spectrum when the oscillator experiences a lateral vibration of $15 \mathrm{~g}(\mathrm{rms})$ at $200 \mathrm{~Hz}$. From plots like Fig. 11, the heights of sideband peaks were determined and the acceleration sensitivity, $\Gamma$, extracted using (3). A statistically relevant set of data was obtained by exciting the oscillator in both lateral and vertical directions with various acceleration amplitudes at $200 \mathrm{kHz}$ and $300 \mathrm{kHz}$.

Fig. 12 summarizes the measured acceleration sensitivity values, which are seen to vary very little with respect to the 


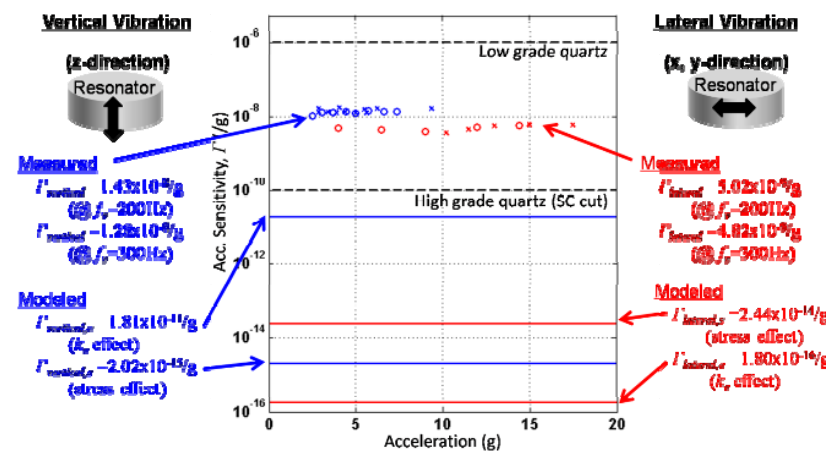

Fig. 12: Summary of measured acceleration sensitivity values for the sub$100 \mathrm{~nm}$ capacitively transduced surface micromachined resonator oscillator. From the data, the micromechanical resonator oscillators of this work perform on par with mid-range commercial quartz-based oscillators. However, theoretical models predict much lower acceleration sensitivities than measured here, which suggests that components other than the resonator in the oscillator circuit might dominate among frequency shifting mechanisms.

vibration frequencies or the acceleration amplitudes. The average values of vertical and lateral acceleration sensitivity for the disk array-composite resonator-based oscillators were measured to be $\Gamma_{\text {verticar }} \sim 13.6 \mathrm{ppb} / \mathrm{g}$ and $\Gamma_{\text {latera }} \sim 4.92 \mathrm{ppb} / \mathrm{g}$, respectively. As indicated in Fig. 12, these preliminary results indicate that the acceleration sensitivity of oscillators referenced to sub-100nm gap capacitively transduced micromechanical resonators are at least on par with mid-grade commercial quartz-based oscillators.

\section{DISCUSSION}

Although the measured acceleration sensitivities are quite good, they are several orders of magnitude higher than those expected by the models of Section III. This suggests the existence of other dominant acceleration-induced mechanisms in the tested oscillator. As discussed in Section II, non-resonant circuit components, such as wire-bonds or soldered items, can often dominate the oscillator acceleration sensitivity. For example, oscillators constructed of only electronic components, i.e., not mechanically resonant ones, have exhibited very high acceleration sensitivities in the range of $\Gamma=10^{-6} \sim 10^{-7} / \mathrm{g}[19]$. A quick glance at Fig. 10b reveals that the rudimentary circuit board used in this study has several potential sources which could easily degrade the acceleration sensitivity of the oscillator. In particular, unbound wires were used to reconfigure the circuit board during testing, and these were probably very susceptible to vibrations. Even if these wires were held down (e.g., via epoxy), there are still aluminum wire-bonds connecting the resonator to the oscillator circuit. Given that wirebonds have acceleration sensitivities high enough to be used even as acceleration sensors in previous work [20], wirebonds could very well limit the acceleration sensitivity resolution of the test apparatus.

To remedy the above issues and allow measurement of the true acceleration sensitivity of micromechanical resonatorbased oscillators, single-chip integration is likely needed [21]. Work towards this is in progress.

\section{CONCLUSIONS}

This work modeled and measured the acceleration sensitivity of oscillators referenced to extremely small gap capa- citvely transduced micromechanical resonators, yielding values for acceleration sensitivity of $\Gamma_{\text {verticar }} \sim 13.6 \mathrm{ppb} / \mathrm{g}$ and $\Gamma_{\text {latera }} \sim 4.92 \mathrm{ppb} / \mathrm{g}$, for directions perpendicular to and parallel to the substrate, respectively. Although on par with commercial quartz-based oscillator products, the measured values are still far higher than the $0.0181 \mathrm{ppb} / \mathrm{g}$ predicted by theory, when assuming electrical stiffness and mechanical stresses as major mechanisms for acceleration sensitivity. Non-resonant board-level components, such as reconfiguring wires and bond wires, are suspected as limiting sources of acceleration sensitivity in this work. Work to fully integrate micromechanical resonator oscillators on single-chips, so as to remove the board and its acceleration sensitive components, is presently underway with the aim of measuring the true acceleration insensitivity achievable via MEMS technology.

\section{ACKNOWLEDGMENT}

This work was supported by the DARPA S\&T Fundamentals program. The authors would especially like to thank Dr. John Vig and Dr. Yoonkee Kim for their advice, plus Dr. Roy Olsson and Dr. Ken Wojciechowski from Sandia National Laboratories for valuable discussions. We would also like to thank Professor Thomas Kenny at Stanford University for use of his shaker in the experimental setup.

\section{REFERENCES}

[1] C. T.-C. Nguyen, "MEMS technology for timing and frequency control," IEEE Trans.on UFFC, vol. 54, pp. 251-70, 2007.

[2] B. Kim, et. al., "Frequency Stability of Wafer-scale Film Encapsulated ...," Sensors and Actuators, A: Physical, vol. 136, pp. 125-131, 2007.

[3] J. C. Salvia, et al., "Real-Time Temperature Compensation of MEMS Oscillators Using ...," J of MEMS vol. 19, pp. 192-201, 2010.

[4] J. Wang, et. al., "1.51-GHz polydiamond $\mu$ mechanical disk resonator with impedance-mismatched support," in MEMS2004, pp. 641-664.

[5] D. Weinstein and S. A. Bhave, "Internal Dielectric Transduction of a 4.5 GHz Silicon Bar Resonator," in IEDM 2007, pp. 415-418.

[6] Y.-W. Lin, et. al., "Low phase noise array-composite micromechanical wine-glass disk oscillator," in IEDM 2005, pp. 287-290.

[7] L.-W. Hung, et. al., "Capacitive transducer strengthening via ALDenabled partial-gap filling," in Hilton Head 08, pp. 208-211.

[8] J. W. Weigold, et. al., "Design and fabrication of submicrometer, single crystal Si accelerometer," J. MEMS vol. 10, pp. 518-524, 2001.

[9] J. R. Clark, et. al., "UHF high-order ...," in FCS 2003, pp. 802-809.

[10] M. Akgul, et. al., "Capacitively Transduced $\mu$ mechanical Resonators with Simultaneous Low $\mathrm{R}_{\mathrm{x}}$ and Q $>70,000$," in $H H$ 2010, pp. 467-470.

[11] R. L. Filler, "The acceleration sensitivity of quartz crystal oscillators: a review," IEEE Trans. on UFFC, vol. 35, pp. 297-305, 1988.

[12] M. Agarwal, et. al., Appl. Phys.Lett., vol. 90, p. 3, Jan 2007.

[13] K. E. Wojciechowski, et. al., "Low Vibration Sensitivity MEMS Resonators," in IEEE FCS, Geneva, Switzerland, 2007, pp. 1220-1224.

[14] A. Hati, et. al.,"Vibration Sensitivity of ..," in FCS 2007, pp. 541-546.

[15] D. S. Steinberg, Vibration Analysis for Electronic Equipment, 3 ed. New York: Wiley-Interscience, 2000.

[16] M. M. Driscoll and J. B. Donovan, "Vibration-Induced Phase Noise: It Isn't Just About the Oscillator," in FCS2007, pp. 535-540.

[17] Y.-W. Lin, et. al.,"Series-resonant VHF micromechanical resonator reference oscillators," IEEE J. of SSC, vol. 39, pp. 2477-2491, 2004.

[18] M. A. Abdelmoneum, et. al., "Stemless wine-glass-mode disk micromechanical resonators," in IEEE MEMS 2003, pp. 698-701.

[19] A. Brannon, et. al., "A Local Oscillator ...," in FCS 2006, pp. 443-447.

[20] Y. T. Liao, et. al.,"A CMOS accelerometer using bondwire inertial sensing," in VLSI Circuits, 2009 Symposium on, 2009, pp. 64-65.

[21] W.-L. Huang, Z. Ren, et. al., "Fully monolithic CMOS nickel micromechanical resonator oscillator," in IEEE MEMS 2008, pp. 10-13. 